\title{
Sea Urchin Pigments as Potential Therapeutic Agents Against the Spike Protein of SARS-CoV-2 Based on in Silico Analysis
}

Elena Susana Barbieri, Tamara Rubilar, Ayelén Gázquez, Marisa Avaro, Erina Noé Seiler, Mercedes Vera-Piombo, Agustín Gittardi, Florencia Chaar, Jimena Pía Fernandez, Lucas Sepulveda

Submitted date: $26 / 06 / 2020$ - Posted date: 29/06/2020

Licence: CC BY-NC-ND 4.0

Citation information: Barbieri, Elena Susana; Rubilar, Tamara; Gázquez, Ayelén; Avaro, Marisa; Seiler, Erina Noé; Vera-Piombo, Mercedes; et al. (2020): Sea Urchin Pigments as Potential Therapeutic Agents Against the Spike Protein of SARS-CoV-2 Based on in Silico Analysis. ChemRxiv. Preprint. https://doi.org/10.26434/chemrxiv.12568595.v1

Several studies have been published regarding the interaction between the spike protein of the novel coronavirus SARS-CoV-2 and ACE2 receptor in the host cells. In the presente work, we evaluated the in silico properties of two sea urchin pigments, Echinochrome A (EchA) and Spinochromes (SpinA) against the Spike protein $(S)$ towards finding a potential therapeutic drug against the disease caused by the novel coronavirus (COVID-19). The best ensemble docking pose of EchaA and SpinA showed a binding affinity of -5.9 and -6.7 kcal mol-1, respectively. The linked aminoacids (T505, G496 and Y449 for EchA and Y449, Q493 and G496 for SpinA) are in positions involved in ACE2 binding in both RBDs frim SARS-CoV and SARS-CoV-2 suggesting that EchA and SpinA may interact with Spike proteins drom both viruses. The results suggest that these pigments could act as inhibitors of $S$ protein, pointing them as antiviral drugs for SARS-CoV-2.

File list (5)

Fig_2B_Spike_RBM_SpinA.pdf (88.37 KiB) view on ChemRxiv - download file Barbieri et al_PreprintSpike_2020.pdf (288.13 KiB) view on ChemRxiv • download file Fig_2A_Spike_RBM_EchA.pdf (87.25 KiB) view on ChemRxiv - download file

Fig_1B_Spike_RBM_SpinA.pdf (85.58 KiB) view on ChemRxiv - download file Fig_1A_Spike_RBM_EchA.pdf (85.69 KiB) view on ChemRxiv - download file 


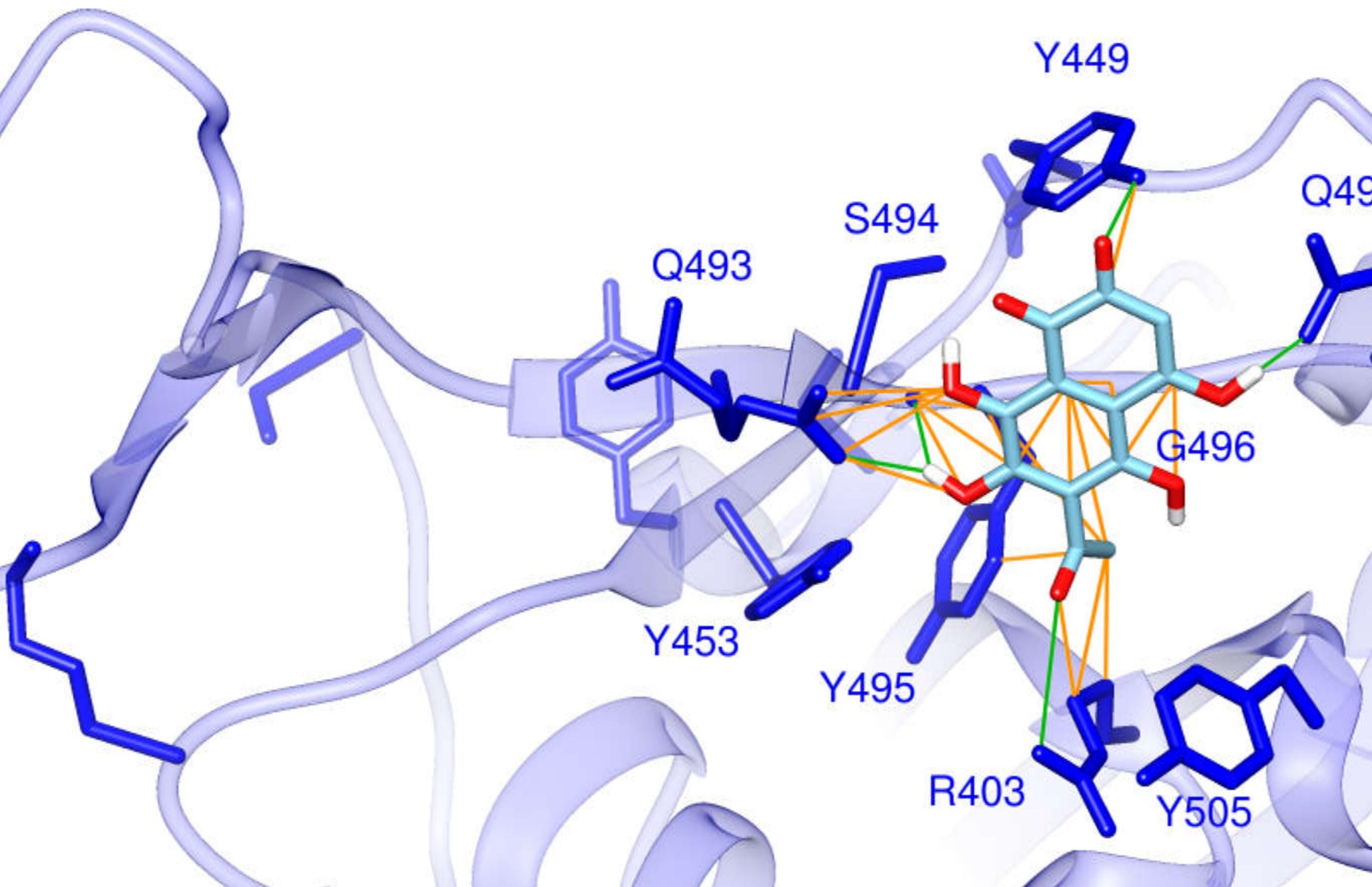


$1 \quad$ Sea urchin pigments as potential therapeutic agents against the spike protein

\title{
of SARS-CoV-2 based on in silico analysis.
}

\author{
Barbieri Elena S. ${ }^{2,3 * a}$, Rubilar Tamara ${ }^{1,3 *}$, Gázquez Ayelén ${ }^{4}$, Avaro Marisa ${ }^{1}$, Seiler, Erina \\ Noé$^{2,3}$, Vera-Piombo Mercedes ${ }^{1,3}$, Gittardi Agustín ${ }^{1}$, Chaar, Florencia ${ }^{1}$, Fernández Jimena \\ Pia $^{1,3}$, Sepúlveda Lucas ${ }^{1,3}$
}

*Both authors contributed equally to the manuscript

${ }^{1}$ Laboratorio de Química de Organismos Marinos - Instituto Patagónico del Mar-UNPSJB, Puerto Madryn, Chubut, Argentina

${ }^{2}$ Laboratorio de Virología - Instituto Patagónico del Mar-UNPSJB, Puerto Madryn, Chubut, Argentina

${ }^{3}$ Laboratorio de Oceanografia Biológica - CESIMAR -CONICET, Puerto Madryn, Chubut, Argentina

${ }^{4}$ Instituto Tecnológico de Chascomú, InTeCh - CONICET, Chascomús, Buenos Aires, Argentina

a- Corresponding author barbieri@,cenpat-conicet.gob.ar - elesuba14@gmail.com

Postal address: Boulevard Brown 2915, Puerto Madryn (9120), Chubut, Argentina.

\begin{abstract}
$\underline{\text { Abstract }}$
In the last few months, several studies have been published regarding the interaction between the Spike protein of the novel coronavirus SARS-CoV-2 and ACE2 receptor in the host cells. In the present work, we evaluated the in silico properties of two sea urchin pigments, Echinochrome A (EchA) and Spinochromes (Spin A) against Spike protein (S) towards finding a potential therapeutic drug against the disease caused by the novel coronavirus (COVID-19). The best ensemble docking pose of EchA and SpinA showed a binding affinity of -5.9 and -6.7 kcal mol ${ }^{-1}$, respectively. The linked aminoacids (Y505, G496 and Y449 for EchA and Y449, Q498, Q493 and G496 for SpinA), are in positions involved in ACE2 binding in both RBDs from SARS-CoV and SARS-CoV-2 suggesting that EchA and SpinA may interact with Spike proteins from both viruses. The results suggest that these pigments could act as inhibitors of $S$ protein, pointing them as antiviral drugs for SARS-Cov-2. Since this study is performed computationally, it requires in vitro and in vivo experiments for further validation.
\end{abstract}

Keywords: 2019 pandemic, 1,4-naphtoquinones polihydroxilate, Echinochrome A, Spinochromes, antiviral drug. 
$\underline{\text { Introduction }}$

Coronaviruses include a wide range of hosts that infect mammalian and avian species. These viruses comprise a large and diverse family of enveloped, positive-stranded RNA viruses. Worldwide, three betacoronaviruses have crossed the species barrier and produced deadly pneumonia in humans. Despite this, the infection by human coronavirus was not considered serious to be controlled by vaccination or to devise specific antivirals until the emergence of severe acute respiratory syndrome (SARS) in 2003 (De Clercq, 2004). Two strains of SARS have generated epidemics: severe acute respiratory syndrome coronavirus (SARS-CoV) and, Middle-East respiratory syndrome coronavirus (MERS-CoV), but it is the current strain that is globally important as a pandemic situation (SARS-CoV-2).

The SARS-CoV-2 disease, COVID-19, has already cost near 479K lives and more than 9 million people are positive confirmed all over the world (https://covid19.who.int/, June $25^{\text {th }}$ 2020). This pandemic is evidence of the potential of coronaviruses to continuously evolve in wild reservoirs and jump to new species (Jaimes et al., 2020).

In this global scenario, there is an urgent requirement for a specific antiviral drug against virus infection and finding the most efficient antiviral drugs available to treat or prevent the disease concerned. New demands for antiviral strategies have increased markedly. The lack of available therapies and vaccines for COVID-19 treatment has led to use of several unsuccessful treatments from drug repositioning of antivirals unable to prevent the death or recovery of patients who ended up with serious lung and heart failures (Guan et al., 2020; Wang et al., 2020; Xu et al., 2020; Zheng et al., 2020; Wang et al., 2020).

The huge variations in host range and tissue tropism among coronaviruses are largely attributable to changes in the homotrimeric spike glycoprotein, liable for binding to the cellular receptors. The spike protein $(\mathrm{S})$ that protrudes from the envelope of the virion, becomes a potential target for vaccines and therapeutic design, as it mediates viral entry into host cells and membrane fusion ( $\mathrm{Li}, 2016$; Tortorici et al., 2019). Spike residues in the viral envelope are responsible for membrane fusion by engaging angiotensin-converting enzyme 2 (ACE2) receptors. ACE2 is found in the heart, lungs, kidney, endothelium, and intestine (Chen et al., 2020; Zhang et al., 2020). Protein S, a trimeric class I fusion protein, is composed of two subunits, $\mathrm{S} 1$ (which contains a receptor binding domain or RBD), responsible for binding to the 
ACE2 receptor on the host cell and S2 (which mediates viral-membrane fusion through the exposure of a highly conserved fusion peptide). Analysis of experimental structures of the SARS-CoV-2 S protein RBD in complex with ACE2 showed that this interface represents an active area of research for therapeutic development (Zhang et al., 2020). Residues on RBD in S protein, essential for ACE2 binding, are highly conserved or share similar side chain properties between SARS-CoV and SARS-Cov-2 which indicate convergent evolution between both RBDs, for improved binding to ACE2 (Lan et al., 2020).

Until date no treatment has been effective in any of these strategies. In order to interfere with key protein required for viral entry into cells and to neutralize essential proteins in viral replication, molecules capable of reaching strategic binding-sites that sometimes are inaccessible to others, are needed. For example, to prevent the virus from entering the cell it is necessary to avoid the successful union of the S protein and the ACE2 receptor by using small molecules (Zhang et al., 2020). The binding of potential small molecules to spike protein can possibly inhibit the replication and transcription of the virus (Rout et al., 2020). In addition to different drug compounds, researchers also look for natural molecules having antiviral activity (Rout et al., 2020).

Among the small molecules, sea urchin pigments are a very interesting group of bioactive compounds that not only have antiviral and antibacterial properties but also reduce ROS stress (Cirino et al., 2017; Fedoreyev et al., 2018). One of the relevant families of sea urchin pigments is 1,4-naphtoquinones polihydroxilate (PHNQs), which includes Spinochrome A and Echinochrome A (Cirino et al., 2017; Fedoreyev et al., 2018; Hou et al., 2020; Vasileva et al., 2017). Specifically, Echinochrome A (EchA) is the active compound of Histochrome ${ }^{\circledR}$ and Gistochrome ${ }^{\circledR}(\mathrm{xx})$, two Russian preparations for cardiopathies and glaucoma diseases that reached the pharmaceutical market and passed all the regulatory requirements. Due to their particular molecular structure, PHNQs pigments possess important antioxidant actions, although their antimicrobial, anti-inflammatory, ion chelating, antiallergic, antidiabetic, antihypertensive, cardioprotective and hypocholesterolemic properties are also highlighted (Jeong et al., 2014; Lebedev et al., 2005; Lennikov et al., 2014; Shikov et al., 2018). The pharmacological activity observed in patients with various alignments, together with the identified low toxicity profiles, strongly support the potential and therapeutic benefits of these natural pigments for the treatment of various human diseases, particularly inflammation, cardioprotection and diabetes (Shikov et al., 2018). One of the computational tools, molecular 
docking, has gained attention as an essential one to investigate potential inhibitor molecules (Rout et al., 2020).

Hence, considering the urgent requirement for a specific antiviral drug, this study aims to examine the in silico properties of EchA and SpinA against Spike protein of SARS-CoV-2, and in this way, suggest a potential therapeutic drug especially against COVID-19, and other coronaviruses as well.

\section{$\underline{\text { Materials and Methods }}$}

In silico study was performed to evaluate the interaction between Echinochrome A (EchA) and Spinochrome A (SpinA) against the viral glycoprotein Spike.

The receptor preparation was done according to Forli et al. (Forli et al., 2016) with a few modifications. SARS-CoV-2 receptor-binding domain (RBD) of the Spike protein cocrystalized with ACE2 (6M0J, resolution $2.45 \AA$ ) was downloaded from RCBS (https://www.rcsb.org/). Water and ligand molecules were removed from the file and the software AutoDockTools (ADT version 1.5.7) was used for receptor preparation. Polar hydrogens were added and partial Kollman charges were assigned. The prepared structures were individually saved in .pdbqt format.

The SMILE of EchA and SpinA were downloaded from Chemical Entities of Biological Interest (ChEBI) and PubChem (https://pubchem.ncbi.nlm.nih.gov/) and, transformed to PDB.

The docking simulations were performed using AutoDock vina 1.1.2 (Trott and Olson, 2010). The center of the search space size for Spike protein docking (-29.04, 30.288, 7.61; $37.50 \times 47.25 \times 46.50 \AA$ ) were set to cover the receptor-binding motif (Lan et al., 2020).

The exhaustiveness has been set to 24 while remaining of AutoDock Vina parameters have been kept at default values. The results of the docking experiment were ranked according to their Vina score and docking poses were visually inspected with UCSF Chimera software (Pettersen et al., 2004). The top ranked candidates were selected for further analysis of proteinligand interactions. Hydrogen bonds (H-bonds) were detected with UCSF Chimera relax Hbonds constraints $\left(0.5 \AA\right.$ and $\left.25^{\circ}\right)$. All direct interactions were also identify as clashes and 
contact. Note that clashes are unfavorable interactions where atoms are too close together while contacts denote all kinds of direct interactions (polar and nonpolar, favorable and unfavorable) including clashes.

\section{$\underline{\text { Results and discussion }}$}

The auto dock software was used for molecular docking analyses of EchA and SpinA against the receptor-binding domain (RBD) complex of the spike protein. The docking consisted of positioning ligands (EchA and SpinA) into the active site and predict how aminoacids will interact in the binding site of the receptor. This technique helps to enhance the success rate of an experiment and cuts down the experimental cost. The molecular docking study can help to analyze the possible binding pose of a small molecule on the active site of a macromolecule (Rout et al., 2020).

The best ensemble docking pose of EchA and SpinA showed a binding affinity of -5.9 and -6.7 kcal mol-1, respectively. These Vina docking scores indicate the stability of the complex for both urchin pigment molecules.

Phylogenetic analysis of RBD showed a similarity between SARS-CoV and SARS-CoV-2 (five out of six hotspot aminoacids in SARS-CoV-2 have their equivalent in SARS-CoV) and the importance of the linked-aminoacids in it (Othman et al., 2020). Computer modelling of the interaction between the SARS-CoV-2 RBD and ACE2 has identified some residues potentially involved in this interaction (Lan et al., 2020). Our molecular docking analyses showed that EchA and SpinA formed hydrogen bonds with different aminoacids residing on the RBD of the Spike protein (Fig. 1).

In particular, the interaction between Spike and EchA (Fig. 2-A) showed three H-bonds with Y449 and Q498 (in green), and 32 Van der Walls (VdW) contacts with R403, S494, Q498, Q493, Y449, Y505, G496, Y495 (in orange), and no clashes. On the other hand, SpinA (Fig. 2B) showed five H-bonds interacted with R403, Y449, Q498, Q493 and S494. Besides, 32 VdW contacts with Q493, Q498, Y495, R403, S494, Y449 and G496, with no clashes. It is important to mention that linked-aminoacids -i.e., Y449, Q498, Q493, Y505, and G496- are part of the receptor-binding motif (RBM) that interacts directly with ACE2 (Lan et al., 2020). 
175 With regard to the linked aminoacids (Y505, G496 and Y449 for EchA and Y449, Q498, Q493

176 and G496 for SpinA), they are in positions involved in ACE2 binding in both RBDs from SARS

177 CoV and SARS CoV 2 suggesting that EchA and SpinA may interact with Spike proteins from

178 both viruses. From this, we suggest that these sea urchin pigments could become possible

179 antiviral drugs because they may interfere with viral infection through binding to Spike

180 glycoprotein with particular interest for experimental evaluation. Actually, there is an urgent

181 need for secure and effective therapeutic options for SARS-CoV-2 infections; in particular,

182 there is no approved therapy for COVID-19. Taking into account the results obtained in this in

183 silico study, we propose an in vitro analysis for evaluating the effect of these two small natural

184 molecules in coronavirus inhibition. Especially EchA is a natural compound already available

185 for other illnesses, and neither toxicity at test concentration nor adverse reactions were found;

186 in addition, all the clinical regulations have been approved. The results showed in this study are

187 part of a provisional patent under revision.

\section{Conclusion}

Our results suggest that sea urchin pigments, EchA and SpinA, could act as inhibitors of S 


\section{$\underline{\text { References }}$}

Chen, L., Li, X., Chen, M., Feng, Y., Xiong, C., 2020. The ACE2 expression in human heart indicates new potential mechanism of heart injury among patients infected with SARS-CoV2. Cardiovasc. Res. https://doi.org/10.1093/cvr/cvaa078

Cirino, P., Brunet, C., Ciaravolo, M., Galasso, C., Musco, L., Fernández, T.V., Sansone, C., Toscano, A., 2017. The sea urchin arbacia lixula: A novel natural source of astaxanthin. Mar. Drugs. https://doi.org/10.3390/md15060187

De Clercq, E., 2004. Antivirals and antiviral strategies. Nat. Rev. Microbiol. 2, 704-720. https://doi.org/10.1038/nrmicro975

Fedoreyev, S.A., Krylova, N. V., Mishchenko, N.P., Vasileva, E.A., Pislyagin, E.A., Iunikhina, O. V., Lavrov, V.F., Svitich, O.A., Ebralidze, L.K., Leonova, G.N., 2018. Antiviral and antioxidant properties of echinochrome A. Mar. Drugs 16, 1-10. https://doi.org/10.3390/md16120509

Forli, S., Huey, R., Pique, M.E., Sanner, M.F., Goodsell, D.S., Olson, A.J., 2016. Computational protein-ligand docking and virtual drug screening with the AutoDock suite. Nat. Protoc. https://doi.org/10.1038/nprot.2016.051

Guan, W., Ni, Z., Hu, Y., Liang, W., Ou, C., He, J., Liu, L., Shan, H., Lei, C., Hui, D.S.C., Du, B., Li, L., Zeng, G., Yuen, K.Y., Chen, R., Tang, C., Wang, T., Chen, P., Xiang, J., Li, S., Wang, J.L., Liang, Z., Peng, Y., Wei, L., Liu, Y., Hu, Y.H., Peng, P., Wang, J.M., Liu, J., Chen, Z., Li, G., Zheng, Z., Qiu, S., Luo, J., Ye, C., Zhu, S., Zhong, N., 2020. Clinical characteristics of coronavirus disease 2019 in China. N. Engl. J. Med. 382, 1708-1720. https://doi.org/10.1056/NEJMoa2002032

Hou, Y., Carne, A., McConnell, M., Bekhit, A.A., Mros, S., Amagase, K., Bekhit, A.E.D.A., 2020. In vitro antioxidant and antimicrobial activities, and in vivo anti-inflammatory activity of crude and fractionated PHNQs from sea urchin (Evechinus chloroticus). Food Chem. https://doi.org/10.1016/j.foodchem.2020.126339

Jaimes, J.A., André, N.M., Chappie, J.S., Millet, J.K., Whittaker, G.R., 2020. Phylogenetic Analysis and Structural Modeling of SARS-CoV-2 Spike Protein Reveals an Evolutionary Distinct and Proteolytically Sensitive Activation Loop. J. Mol. Biol. 432, 3309-3325. https://doi.org/10.1016/j.jmb.2020.04.009

Jeong, S.H., Kim, H.K., Song, I.S., Lee, S.J., Ko, K.S., Rhee, B.D., Kim, N., Mishchenko, N.P., Fedoryev, S.A., Stonik, V.A., Han, J., 2014. Echinochrome a protects mitochondrial function in cardiomyocytes against cardiotoxic drugs. Mar. Drugs. https://doi.org/10.3390/md12052922

Lan, J., Ge, J., Yu, J., Shan, S., Zhou, H., Fan, S., Zhang, Q., Shi, X., Wang, Q., Zhang, L., Wang, X., 2020. Structure of the SARS-CoV-2 spike receptor-binding domain bound to the ACE2 receptor. Nature 581, 215-220. https://doi.org/10.1038/s41586-020-2180-5

Lebedev, A. V., Ivanova, M. V., Levitsky, D.O., 2005. Echinochrome, a naturally occurring iron chelator and free radical scavenger in artificial and natural membrane systems. Life Sci. https://doi.org/10.1016/j.lfs.2004.10.007

Lennikov, A., Kitaichi, N., Noda, K., Mizuuchi, K., Ando, R., Dong, Z., Fukuhara, J., Kinoshita, S., Namba, K., Ohno, S., Ishida, S., 2014. Amelioration of endotoxin-induced uveitis treated with the sea urchin pigment echinochrome in rats. Mol. Vis.

Li, F., 2016. Structure, Function, and Evolution of Coronavirus Spike Proteins. Annu. Rev. Virol. https://doi.org/10.1146/annurev-virology-110615-042301

Othman, H., Bouslama, Z., Brandenburg, J.T., da Rocha, J., Hamdi, Y., Ghedira, K., Srairi-Abid, N., Hazelhurst, S., 2020. Interaction of the spike protein RBD from SARS-CoV-2 with ACE2: Similarity with SARS-CoV, hot-spot analysis and effect of the receptor polymorphism. Biochem. Biophys. Res. Commun. 527, 702-708. https://doi.org/10.1016/j.bbrc.2020.05.028

Pettersen, E.F., Goddard, T.D., Huang, C.C., Couch, G.S., Greenblatt, D.M., Meng, E.C., Ferrin, T.E., 2004. UCSF Chimera - A visualization system for exploratory research and analysis. J. Comput. Chem. https://doi.org/10.1002/jcc.20084 
Rout, J., Swain, C., Tripathy, U., 2020. In Silico Investigation of Spice Molecules as Potent Inhibitor of SARS-CoV-2. Chemrxive. https://doi.org/10.26434/chemrxiv.12089730.v1 Scope

Shikov, A.N., Pozharitskaya, O.N., Krishtopina, A.S., Makarov, V.G., 2018. Naphthoquinone pigments from sea urchins: chemistry and pharmacology. Phytochem. Rev. https://doi.org/10.1007/s11101-018-9547-3

Tortorici, M.A., Walls, A.C., Lang, Y., Wang, C., Li, Z., Koerhuis, D., Boons, G.J., Bosch, B.J., Rey, F.A., de Groot, R.J., Veesler, D., 2019. Structural basis for human coronavirus attachment to sialic acid receptors. Nat. Struct. Mol. Biol. https://doi.org/10.1038/s41594019-0233-y

Trott, O., Olson, A.J., 2010. AutoDock Vina: Improving the Speed and Accuracy of Docking with a New Scoring Function, Efficient Optimization, and Multithreading. J. Comput. Chem. 31, 455-461. https://doi.org/10.1002/jcc

Vasileva, E.A., Mishchenko, N.P., Fedoreyev, S.A., 2017. Diversity of Polyhydroxynaphthoquinone Pigments in North Pacific Sea Urchins. Chem. Biodivers. https://doi.org/10.1002/cbdv.201700182

Wang, D., Hu, B., Hu, C., Zhu, F., Liu, X., Zhang, J., Wang, B., Xiang, H., Cheng, Z., Xiong, Y., Zhao, Y., Li, Y., Wang, X., Peng, Z., 2020. Clinical Characteristics of 138 Hospitalized Patients with 2019 Novel Coronavirus-Infected Pneumonia in Wuhan, China. JAMA - J. Am. Med. Assoc. https://doi.org/10.1001/jama.2020.1585

Xu, Z., Shi, L., Wang, Y., Zhang, J., Huang, L., Zhang, C., Liu, S., Zhao, P., Liu, H., Zhu, L., Tai, Y., Bai, C., Gao, T., Song, J., Xia, P., Dong, J., Zhao, J., Wang, F.S., 2020. Pathological findings of COVID-19 associated with acute respiratory distress syndrome. Lancet Respir. Med. 8, 420-422. https://doi.org/10.1016/S2213-2600(20)30076-X

Zhang, H., Penninger, J.M., Li, Y., Zhong, N., Slutsky, A.S., 2020. Angiotensin-converting enzyme 2 (ACE2) as a SARS-CoV-2 receptor: molecular mechanisms and potential therapeutic target. Intensive Care Med. https://doi.org/10.1007/s00134-020-05985-9

Zheng, Y.Y., Ma, Y.T., Zhang, J.Y., Xie, X., 2020. COVID-19 and the cardiovascular system. Nat. Rev. Cardiol. 17, 259-260. https://doi.org/10.1038/s41569-020-0360-5 


\section{$280 \quad$ Figure caption}

281 Figure 1. SARS-CoV-2 RBD Spike protein interaction with urchin pigments. (A)

282 Structure of SARS-CoV-2 RBD Spike bound to EchA best docking pose. EchA is shown

283 in green, SARS-CoV-2 RBD Spike core is shown in blue and the RBM is shown in red.

284 (B) Structure of SARS-CoV-2 RBD Spike bound to SpinA best docking pose. SpinA is 285 shown in green, SARS-CoV-2 RBD Spike core is shown in blue and the RBM is shown 286 in red.

287

$288 \quad \mathbf{A}$

289

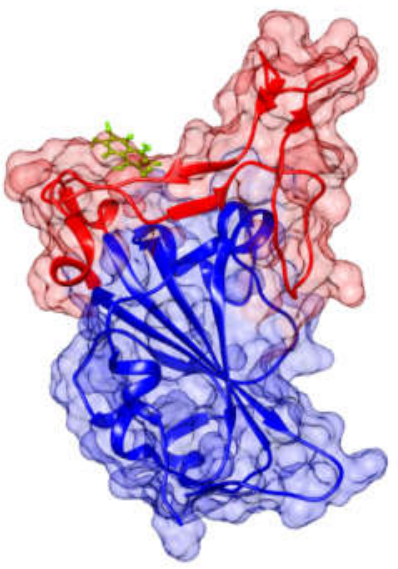

290

291

292

B

293

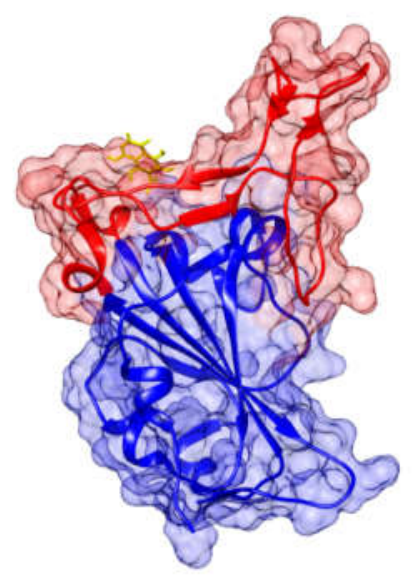


296 Figure 2. Details of H-bonds and Van der Waals interactions between urchin pigments 297 and RBD. (A) Analyzed interactions between EchA best docking pose and SARS-CoV2982 RBD and (B) Analyzed interactions between SpinA best docking pose and SARS-CoV2992 RBD. The H-bonds are highlighted in green, and VdW interactions in orange. Main 300 amino acids participating on the interactions are labeled.

301

302

A

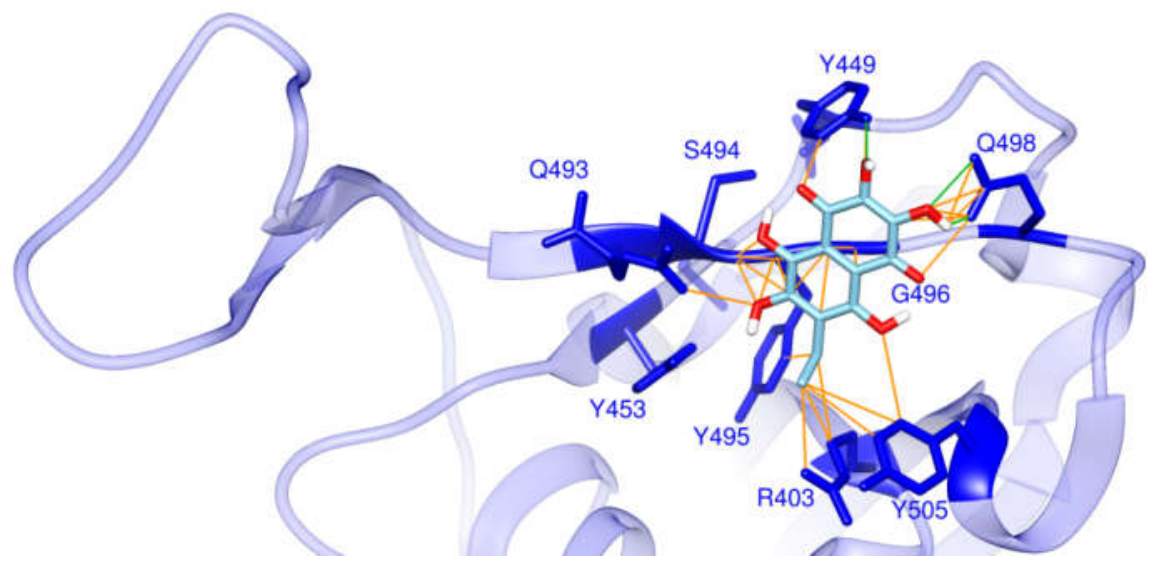

305

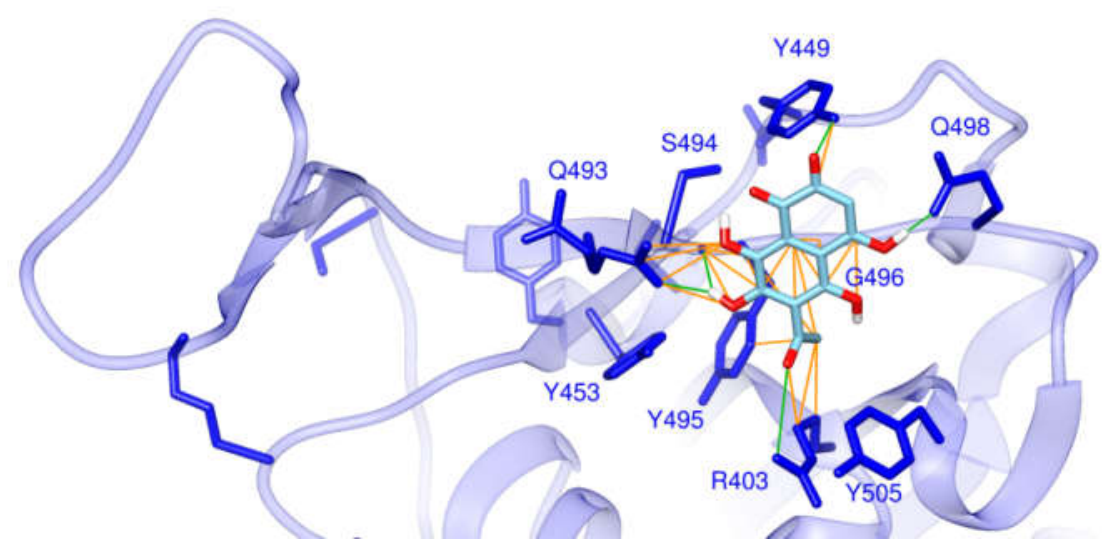




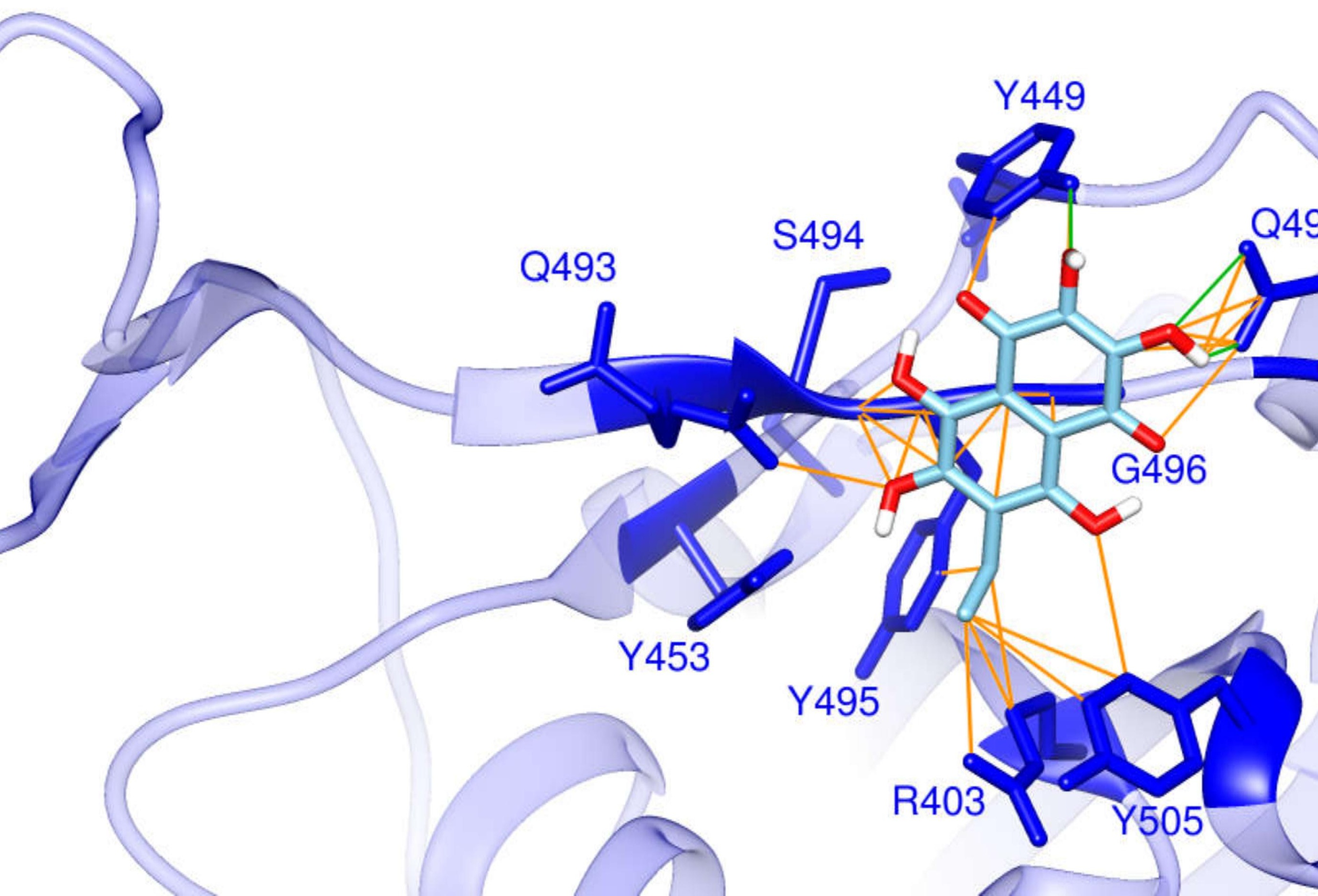




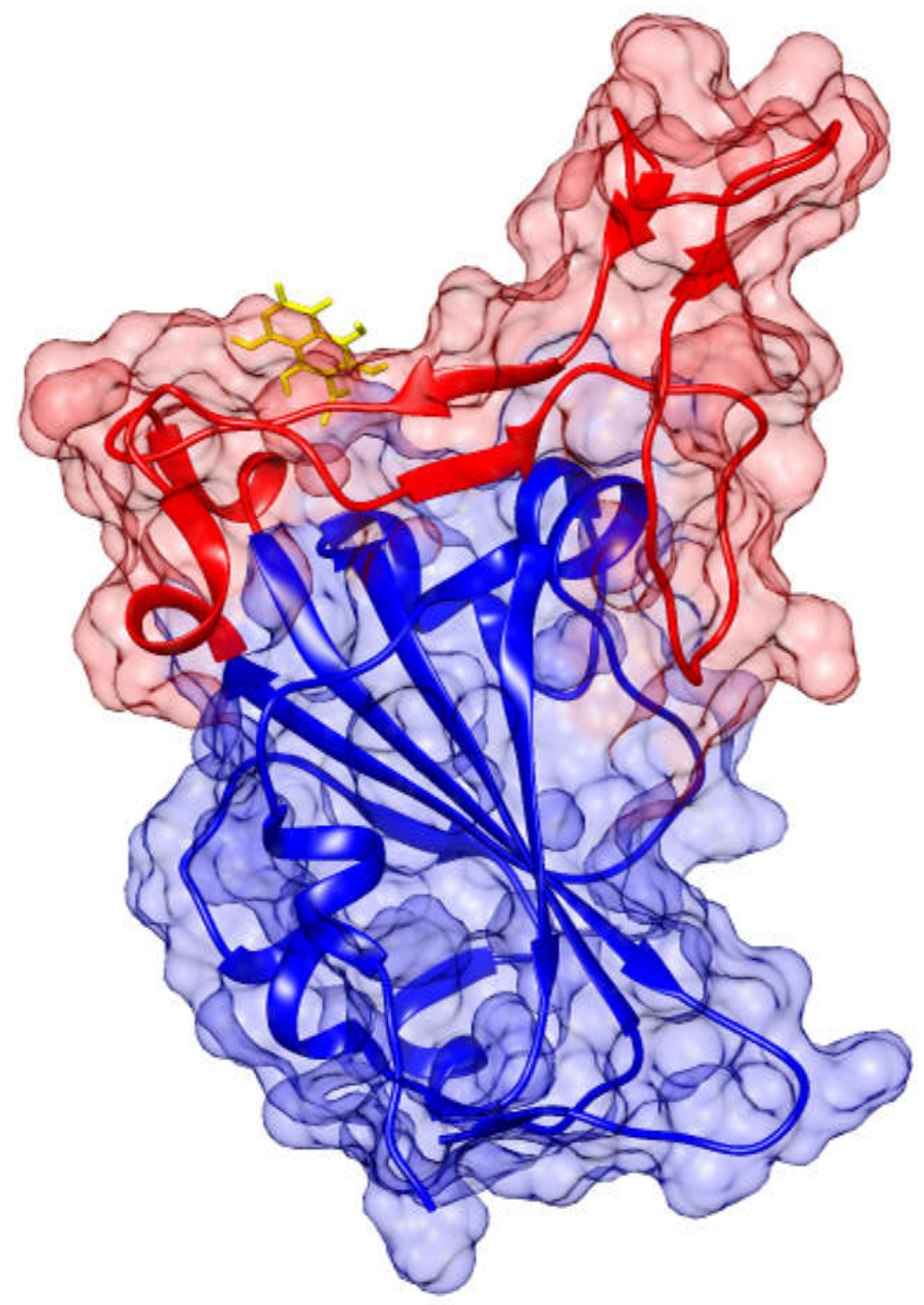




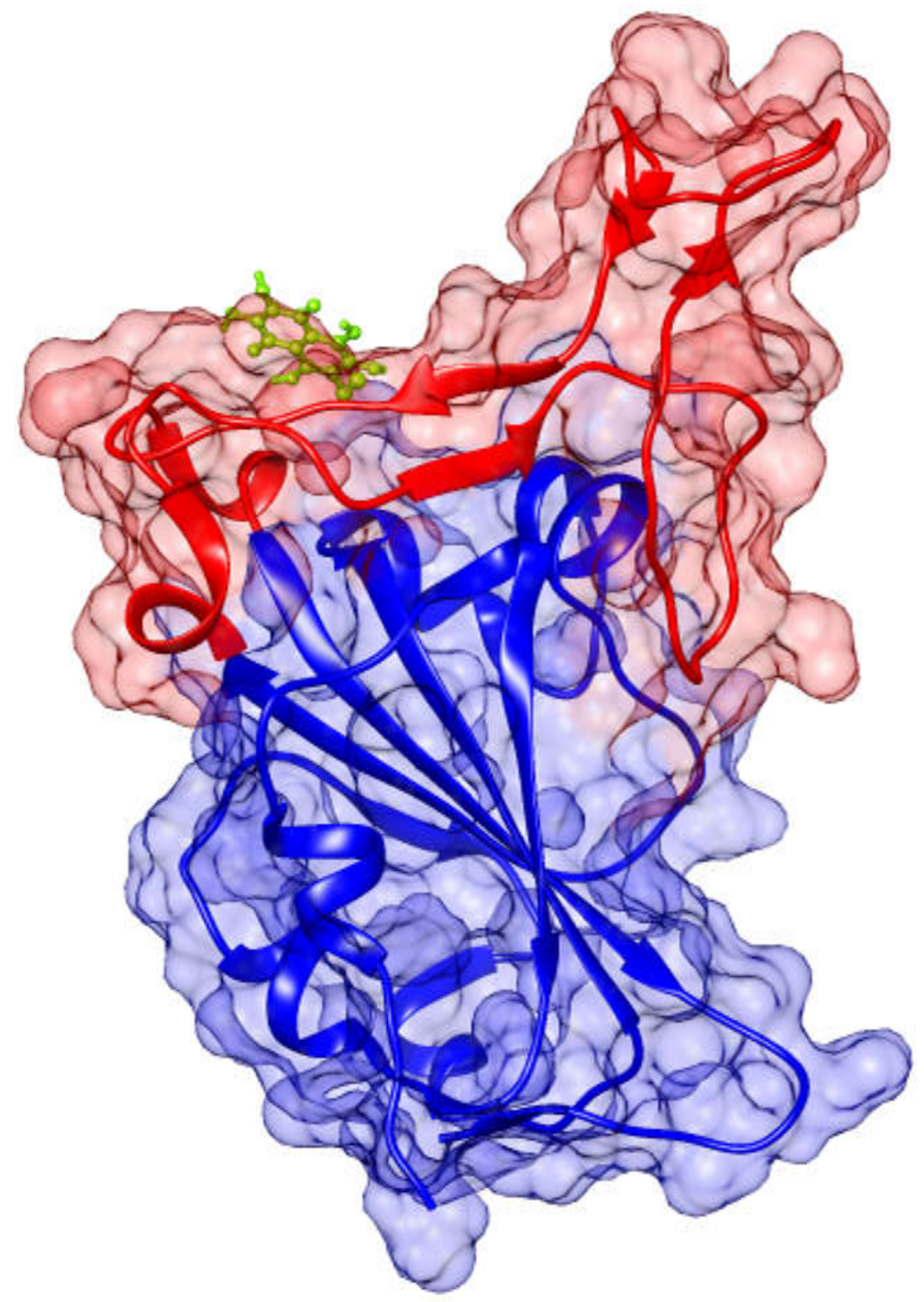


